\title{
Antagonistic effects of intestinal Lactobacillus isolates on pathogens of chicken
}

\begin{abstract}
Twelve Lactobacillus strains isolated from chicken intestine, which demonstrated a strong and moderate capacity to adhere to the ileal epithelial cells in vitro, were used to investigate their inhibitory ability against five strains of salmonella, i.e. Salmonella enteritidis 935/79, Salm. pullorum, Salm. typhimurium, Salm. blockIey and Salm. enteritidis 94/448, and three serotypes of Escherichia coli, viz. E. coli O1: K1, O2: K1 and O78: K80. The results showed that all the 12 Lactobacillus isolates were able to inhibit the growth of the five strains of salmonella, and the three strains of E. coli in varying degrees. Generally, they were more effective in inhibiting the growth of salmonella than E. coli. Inhibition of the pathogenic bacteria was probably due to the production of organic acids by the Lactobacillus isolates.
\end{abstract}

Keyword: Lactobacillus; Chicken intestine; Salmonella; Inhibitory ability 